CHERCHER, REPÉRER, AVANCER

\title{
QUAND L'ÉTAT FAIT SON MARCHÉ, QUELS EFFETS POUR LES OPÉRATEURS ?
}

Genèse et mise en œuvre de la sous-traitance dans la politique de l'emploi (1945-2014)

Thierry Berthet, Clara Bourgeois

Presses de Sciences Po (P.F.N.S.P.) | « Gouvernement et action publique »

$2017 / 4 N^{\circ} 4 \mid$ pages 75 à 99

ISSN 2260-0965

ISBN 9782724634983

Article disponible en ligne à l'adresse :

https://www.cairn.info/revue-gouvernement-et-action-publique-2017-4-page-75.htm

\section{Pour citer cet article :}

Thierry Berthet, Clara Bourgeois« Quand l'État fait son marché, quels effets pour les opérateurs ? Genèse et mise en œuvre de la sous-traitance dans la politique de l'emploi (1945-2014) », Gouvernement et action publique 2017/4 (N² 4), p. 75-99. DOI 10.3917/gap.174.0075

Distribution électronique Cairn.info pour Presses de Sciences Po (P.F.N.S.P.).

(C) Presses de Sciences Po (P.F.N.S.P.). Tous droits réservés pour tous pays.

La reproduction ou représentation de cet article, notamment par photocopie, n'est autorisée que dans les limites des conditions générales d'utilisation du site ou, le cas échéant, des conditions générales de la licence souscrite par votre établissement. Toute autre reproduction ou représentation, en tout ou partie, sous quelque forme et de quelque manière que ce soit, est interdite sauf accord préalable et écrit de l'éditeur, en dehors des cas prévus par la législation en vigueur en France. Il est précisé que son stockage dans une base de données est également interdit. 


\title{
QUAND L'ÉTAT FAIT SON MARCHÉ, QUELSEFFETS POUR LES OPÉRATEURS ?
}

\author{
Genèse et mise en œuvre de la sous-traitance \\ dans la politique de l'emploi (1945-2014)
}

\section{Thierry Berthet ${ }^{1}$, Clara Bourgeois}

Résumé : À partir de l'après-guerre, le placement des demandeurs d'emploi est un monopole d'État confié à une agence publique, l'Agence nationale pour l'emploi (ANPE). Le milieu des années 2000 a été le moment d'un tournant qui opère une brèche dans ce monopole en amplifiant le recours à des quasi-marchés de prestataires privés. En dépit d'une sémantique qui fait une place centrale à la notion de " marché » du travail et de l'emploi, cet article s'attachera à démontrer que la régulation des prestations d'accompagnement et de placement offertes aux chômeurs demeure entre les mains de l'opérateur public. L'analyse de la genèse de la sous-traitance dans la politique de l'emploi permet de révéler que ce phénomène obéit davantage à des impératifs contextuels qu'à une perspective stratégique globale et qu'il demeure marginal. De plus, l'analyse du fonctionnement concret des marchés de prestations aux chômeurs via les pratiques des prestataires de services permet de mettre en valeur le poids des instruments d'action publique au regard du renforcement des rapports de force entre acteurs de l'insertion professionnelle.

MOTS-CLÉS : EMPLOI - EXTERNALISATION - MARCHANDISATION - OPÉRATEURS DE TERRAIN - PÔLE EMPLOI - SERVICE PUBLIC DE L'EMPLOI

\section{LIBERALIZING THE LABOR MARKET? ORIGINS AND IMPLEMENTATION OF THE FRENCH EMPLOYMENT POLICY'S MARKETIZATION (1945-2014)}

Abstract: Since the post-war period, the placement of jobseekers in France has been a state monopoly entrusted to a public agency, the National Employment Agency (ANPE). The mid-2000s represents a turning point in breaking this monopoly by initiating the use of quasi-market private providers. Despite a semantics that focuses on the notion of "the labor market", this article demonstrates that the employment policy's implementation remains firmly controlled by the public employment service. The chronological analysis of subcontracting in employment policy undertaken here reveals that this phenomenon is more a matter of contextual imperatives than of a marketization strategic perspective which, at best, remains peripheral. Moreover, fieldwork-based analysis of

1. Cet article a été rédigé à l'occasion d'un séjour de recherche à l'École française de Rome. Thierry Berthet tient à remercier la direction de l'École française de Rome et de la section contemporaine pour son accueil. 
the concrete practices of public employment service agents and private providers highlights the weight of public policy instruments in reinforcing pre-existing power relations between the actors specialized in social insertion through work.

\section{KEYWORDS: EMPLOYMENT POLICY - MARKETIZATION - PUBLIC EMPLOYMENT SERVICE - STREET LEVEL BUREAUCRACY - SUBCONTRACTING}

Marché du travail, marché de l'emploi, la métaphore du marché est omniprésente dans le langage commun comme scientifique. Les débats sur l'authenticité de ce marché et la pertinence de cette métaphore sont anciens. Depuis Locke (Diemer, Guillemin, 2009) jusqu'à Polanyi (Postel, Sobel, 2010), on s'interroge sur la réalité du travail comme marchandise. II faut en premier lieu opérer une distinction entre ces débats sur la nature du travail et ceux, liés aux évolutions contemporaines des méthodes de gouvernement, qui portent sur la mise en marché des politiques publiques et singulièrement ici de celles visant l'accompagnement vers l'emploi. C'est à ce deuxième pan que s'adresse cet article qui vise à apporter une contribution sectorielle à l'analyse de la mise en marché de l'action publique.

Les politiques d'emploi représentent un champ dont la régulation a historiquement constitué, comme le rappelle Pierre Muller (2015, 32sq.), I'un des creusets du développement des politiques publiques et de leur sectorisation. Le recours croissant aux opérateurs privés et à la mise en marché de l'intervention publique constitue une modalité de plus en plus fréquente de mise en œuvre des politiques publiques notamment dans le champ des politiques sociales (Van Berkel et al., 2012). On pourrait ainsi s'attendre à ce que la régulation publique du secteur de l'emploi s'appuie fortement sur de telles logiques (Considine, 2001; Zimmermann et al., 2014). Pourtant, cette métaphore « dangereuse » (Galbraith, 1997) du marché n'est pas seulement hasardeuse en termes de théorie des échanges économiques, elle est également récente et faiblement significative en tant que principe d'action publique sectoriel dans le champ des politiques d'emploi. L'hypothèse que nous avançons ici à cet égard est que le recours à la forme du marché s'incarne davantage dans une « régulation concurrentielle subventionnée » (Vatan, 2013, p. 3 ; Cuntigh et al., 2008) où les opérateurs publics conservent la main que dans une privatisation de l'action publique (Ansaloni et Smith en introduction à ce dossier).

Les politiques de l'emploi sont l'objet de vifs débats, y compris au sein des composantes du Service public de l'emploi (SPE). Ils portent notamment sur la « mise en marché » de cette politique publique. La vivacité de ces controverses est réactivée de manière cyclique durant les périodes de crise, comme celle ouverte depuis 2008, qui attisent débats et propositions de « nouvelles solutions " pour " résoudre la crise du chômage ". Parmi celles ci, la contestation d'une régulation purement étatique et la mise en concurrence des opérateurs en charge de l'accompagnement des demandeurs d'emploi apparaît comme un changement de paradigme ${ }^{2}$ nécessaire. L'adoption d'une perspective diachronique permet de retracer de manière synthétique l'émergence de formes de régulation marchande dans la politique de l'emploi. Une

2. Depuis les travaux de Hall sur les ordres de changement (Hall, 1993), il est généralement entendu en analyse des politiques publiques qu'un changement de paradigme suppose une modification substantielle des instruments, des configurations d'acteurs et des objectifs d'une politique publique (Muller, Surel, 1998). 
telle sociogenèse de l'externalisation vise à mettre en évidence une énigme liée à la diffusion et l'intensité des changements introduits. D'un côté, un paradigme orienté par la marchandisation - entendue ici comme une régulation fondée sur la mise en concurrence des opérateurs - apparaît avec ses nouveaux acteurs et des instruments inédits. De l'autre, sa diffusion demeure restreinte, voire régresse et a pour effet de renforcer le rôle et la position des opérateurs publics. Qui plus est, les motifs de la mise en marché et ses modalités demeurent largement, pour l'administration de l'emploi, de nature technique et ne sont pas innervés par un répertoire de légitimation emprunté aux principes de la " nouvelle gestion publique » (Merrien, 1999). La mise en concurrence de l'opérateur public (ANPE) opérée par l'Unedic dès le début des années 2000 se solde ainsi par un ex aequo, voire un léger avantage à l'ANPE et l'argument d'efficience accrue du marché a été de la sorte délégitimé d'entrée de jeu; la fusion ANPE/Unédic ayant au final réglé les différends en prolongeant les pratiques d'externalisation sous un contrôle strict du nouvel opérateur public, Pôle emploi.

Cette mise en perspective diachronique permet ainsi d'interroger, et ultimement de nuancer, la logique de "marchandisation » de l'action publique en matière d'emploi. Par marchandisation, on entendra ici une forme d'instrumentation de l'action publique fondée sur le recours systématique aux mécanismes de marché compris comme " concurrence entre acteurs publics et avec le secteur privé, individualisation des incitations, externalisation de l'offre » (Bezes et al., 2011). Ainsi définie comme un processus centré sur la mise en concurrence systématique des opérateurs (Greer, Doellgast, 2017)3, la mise en marché de l'accompagnement des demandeurs d'emploi sera discutée dans la première partie de ce texte au prisme des éléments d'analyse (participants, attributs de la marchandise, modes d'organisation des transactions, valeur d'échange de la marchandise) des institutions caractérisant les marchés tels que formalisés par Smith et Ansaloni en introduction à ce dossier. Plus précisément, l'objet de cette première partie sera de retracer, en se basant sur une perspective macro analytique, l'émergence de mécanismes de régulation marchande dans la mise en œuvre de la politique de l'emploi. À partir d'une lecture diachronique, elle vise à informer précisément le développement d'un marché d'État (Ansaloni et Smith, introduction à ce dossier) à travers les réformes intervenues dans le champ de l'accompagnement des demandeurs d'emploi.

Dans une deuxième partie de cet article, l'analyse se déplace vers un niveau plus opérationnel et territorial : celui du rôle et des effets sur les agents de terrain, publics comme privés. L'analyse portera sur les pratiques des agents sur la période allant de 2011 à 2014. Leur pratique concrète de la mise en marché de l'accompagnement et du placement des demandeurs d'emploi s'inscrit dans le cadre normatif présenté en première partie. Elle est inspirée par un instrument-clé - le code des marchés publics ${ }^{4}$ - qui définit les règles d'achat et de commande de services ou de biens. II s'adresse à l'État, aux établissements publics, aux collectivités territoriales et enfin aux organismes de sécurité sociale ${ }^{5}$. II s'agit d'une

3. Le processus de marchandisation ainsi décrit place la transaction au cœur de la délivrance du service : «A fully marketized transaction is one with intense price-based competition, in which actor choices are made purely on the basis of price, the good or service in question is standardized, exchanges are frequent, and competition is open to a wide range of participants " (Greer, Doellgast, 2017, p. 195).

4. [www.legifrance.gouv.fr/affichCode.do?cidTexte=LEGITEXT000005627819].

5. Pôle emploi, à l'instar d'autres organismes, n'est pas soumis au code des marchés publics, mais à l'ordonnance de 2005 (ordonnance $n^{\circ}$ 2005-649 du 6 juin 2005) qui repose sur les mêmes principes d'action et correspond à une version simplifiée du code des marchés publics. 
logique de mise en concurrence des fournisseurs qui vise à réguler les relations entre les autorités publiques et les prestataires de service avec lesquelles un contrat est établi en passant d'une logique de subvention à une logique d'achat de prestations (Berthet, 2010, 2012). C'est sur ce niveau opératoire que, dans un second temps, nous porterons le regard pour montrer l'effet de ce cadre normatif sur les interactions entre opérateurs. En effet, la plupart des travaux portant sur l'externalisation et la marchandisation discutent la plus-value d'un recours à un prestataire extérieur en termes d'accompagnement et de résultats en termes de retour à l'emploi (Divay, 2009 ; Parent et al., 2014). En décalage avec ces travaux comme avec ceux abordant la marchandisation de l'action publique sous un angle généraliste, nous proposons d'observer, sur la base d'un travail empirique de sociologie de l'action publique, les effets de la logique de marchés publics sur les relations entre acteurs de l'emploi et les stratégies de résistances à sa mise en œuvre.

Ces deux niveaux d'analyse visent à mettre en relation les deux pôles d'une chaîne d'action publique allant du cadrage du problème de l'emploi à la mise en œuvre des dispositifs concrets où la mise en marché fait figure d'instrument privilégié. À travers cette démarche, nous visons à montrer d'une part que la mise en marché des prestations d'accompagnement des demandeurs d'emploi, si elle a permis l'émergence d'un marché des opérateurs privés de placement, a néanmoins abouti à renforcer la position de l'opérateur public. D'autre part, que si cette ouverture a rigidifié les règles de l'externalisation, il n'en demeure pas moins que les opérateurs de terrain parviennent à se ménager des marges de manœuvre dans la mise en œuvre.

\section{Encadré 1. Sources et méthodes}

La première section de cet article constitue une analyse de synthèse basée sur différents matériaux empiriques accumulés par les auteurs. Ces sources relèvent notamment des éléments suivants: la participation d'un des auteurs à la commission d'évaluation du Commissariat général du plan sur le recours à des opérateurs externes dans la conduite de la politique de l'emploi ; une veille et une analyse documentaire approfondie ; ainsi que des entretiens conduits régulièrement avec des acteurs nationaux de la politique de l'emploi dans le cadre d'une série de programmes de recherche du Céreq conduits par les auteurs durant une décennie au Centre Émile Durkheim. La seconde section de l'article s'appuie sur une large enquête empirique réalisée dans trois villes en France entre 2011 et 2014 dans le cadre d'un projet de recherche européen et d'une thèse de science politique (Bourgeois, 2015). Ce travail s'appuie ainsi sur une importante campagne d'entretiens réalisés entre 2011 et 2013 auprès de conseillers en insertion à Pôle emploi, d'une part, et de conseillers travaillant au sein d'entreprises spécialisées dans le conseil et l'accompagnement des demandeurs d'emploi, de PLIE et de missions locales. Des entretiens ont été également menés auprès de directeurs d'agence Pôle emploi et de directeurs des structures prestataires de service mentionnés précédemment. Au total, 87 entretiens ont été menés dans trois unités urbaines dans le cadre du programme de recherche européen VII PCRD « Localise " ? Local worlds of social cohesion consacré à l'analyse critique d'une approche « intégrée » de l'activation dans les politiques nationales et locales d'emploi. 


\section{Des politiques de l'emploi à leur mise en marché : un changement de paradigme en trois temps}

En un peu plus d'un demi-siècle, les politiques de l'emploi ont considérablement évolué au triple niveau des objectifs poursuivis, des organisations qui les supportent et des instruments permettant leur mise en œuvre. La question du développement d'une régulation marchande de l'emploi interroge et suppose des changements drastiques à ces trois niveaux. Pour en rendre compte, une approche diachronique s'impose. Elle permet de mettre en évidence comment le choix d'un monopole public dans l'immédiat après-guerre a été progressivement remis en cause de manière silencieuse et technique (1945-2005). Cette nouvelle donne, après un processus rapide d'institutionnalisation qui opère un changement radical dans la mise en marché de l'accompagnement des demandeurs d'emploi (2005-2010), va pourtant connaître un ralentissement (2011-2015) qui aboutit au final à renforcer la capacité d'action des opérateurs publics plutôt qu'à l'affaiblir (Le Galès, Scott, 2008).

\section{Du monopole public à la découverte d'un marché (1945-2005)}

Les "politiques de la main-d'œuvre " (c'est le terme consacré à l'époque) comme de nombreux champs des politiques sociales sont "cadrées " dans l'immédiat après-guerre par une ordonnance, celle du 24 mai 1945 relative au placement des travailleurs et au contrôle de l'emploi (Fournier, 2000, p. 5). Ce cadrage dans les grandes ordonnances sociales d'après-guerre contribue à les ancrer dans la nébuleuse des politiques sociales et les lier durablement aux évolutions de l'État-providence. Cette ordonnance et les textes afférents (décret du 23 août et arrêté du 6 octobre 1945) posent comme principes ceux d'un monopole étatique, d'une suppression des bureaux de placement privés payants et du contrôle des embauches/licenciements par l'administration publique. Confié à l'Agence nationale pour l'emploi (ANPE) dès sa création en $1967^{6}$, le monopole public du placement des demandeurs d'emploi demeure la règle jusqu'au milieu des années 2000. Sur la base de ce choix initial, l'ANPE est en charge de l'appariement de la demande et de l'offre d'emploi pour tout le territoire national. Elle exerce sa mission de suivi des demandeurs d'emploi de manière monopolistique et gratuite pour ses usagers. L'ordonnance 86-1286 du 20 décembre 1986 réaffirme ce principe inscrit dès lors à l'article 311-1 dans le code du travail. Sur cette base juridique, les autorités judiciaires, notamment la Cour de cassation ${ }^{7}$, veillent à l'application de ce principe n'hésitant pas à sanctionner les tentatives visant à développer un marché privé et payant du placement.

Pour autant, le monopole de placement de l'ANPE n'empêche pas le développement discret et progressif d'un marché d'opérateurs privés. Mais ce marché est fortement contrôlé

6. Créée par Jacques Chirac en 1967, l'ANPE prend le relais des Services extérieurs du travail et de la maind'œuvre (SETMO) qui avaient « étatisé " en 1946, sous l'égide du ministère du Travail, les offices de placement préexistants (Muller, 1990). La création d'une agence extérieure mais sous tutelle du ministère est ainsi une première forme d'externalisation.

7. À l'exemple de France Personnel, bureau de placement privé, dont la spécificité néerlandophone pour l'industrie hôtelière et touristique semblait dissimuler un placement de main-d'œuvre à bas coût, qui a été sanctionné pour avoir transgressé ce principe (Cour de cassation, Chambre criminelle, 16 novembre 1999, 98-87.686). 
par le premier cercle du Service public de l'emploi (ministère du Travail, ANPE, AFPA) qui y fait régulièrement recours pour faire face à la croissance d'un chômage de masse. C'est ainsi par l'externalisation d'une partie de ses missions que se développe une première forme de marchandisation des activités d'accompagnement et de placement des demandeurs d'emploi. En 2002, une commission d'évaluation du Commissariat général du plan (CGP) est chargée de conduire un premier bilan de ce phénomène d'externalisation. Ce travail évaluatif va conduire à la découverte de l'ampleur insoupçonnée du phénomène. Conduits entre 2002 et 2003, les travaux de cette commission d'évaluation (Balmary et al., 2004) proposent un premier éclairage sur le recours à des opérateurs externes dans la conduite de la politique de l'emploi. Les travaux du CGP dessinent les contours d'un recours massif, parfois ancien, diversement organisé selon les opérateurs et touchant tous les segments de la mise en œuvre de ces politiques (Berthet, 2010). Ce bilan dessine un paysage de la sous-traitance organisé en France autour de trois cercles : l'administration de l'emploi, une série de partenariats institutionnalisés et un marché de la sous-traitance. L'administration de l'emploi se structure alors autour d'un noyau dur composé des services du ministère du Travail, de l'ANPE et de l'AFPA (Association pour la formation professionnelle des adultes) puis d'un cercle élargi aux services des ministères des droits des femmes et de l'action sociale après 1999. Consacrés par le contrat de progrès État/ANPE 1994-1998, des partenariats externes institutionnalisés ont été noués par l'ANPE avec une série d'opérateurs pour prendre en charge l'accès à l'emploi de trois catégories de public : les jeunes (réseau des missions locales), les cadres (APEC) et les personnes handicapées (réseau des Cap Emploi). Ce deuxième cercle fait l'objet d'une externalisation institutionnalisée désignée par l'ANPE sous le terme de " cotraitance ». Les principes de cette cotraitance sont : une coproduction des services, un principe de réciprocité basé sur l'égalité des partenaires et le respect des pratiques de chacun, un conventionnement actualisé de manière régulière (Balmary et al., 2004, p. 155). Dans le cadre de cette délégation, les procédures sont plus souples mais aussi plus floues que les règles des marchés publics qui s'appliquent au troisième cercle, celui des prestataires. II constitue le cœur de pratiques massives d'externalisation. La sous-traitance de l'ANPE est en effet considérable - elle concerne en 2002 entre 70 et 100 \% du volume des prestations d'accompagnement des chômeurs selon le type (Balmary et al., 2004, p. 151) - et repose sur deux principes : la mise en concurrence systématique des opérateurs selon les règles du code des marchés publics et une régulation à la fois hiérarchique et déconcentrée des procédures. La nature des prestations achetées en marché par l'ANPE englobe une palette très large de dispositifs allant du soutien à la création d'entreprise au bilan de compétences approfondi en passant par de nombreuses opérations de préparation et d'intermédiation vers l'emploi. En termes de masses financières, le constat opéré par le CGP est là aussi édifiant, les prestations externalisées en marché de l'ANPE ont vu leur volume augmenter de $759 \%$ entre 1998 et 2002. Elles passent de 45,67 millions d'euros en 1998 à 346,83 millions d'euros en 2002 (Balmary et al., 2004, p. 95).

En termes de logiques d'action, l'externalisation des prestations pratiquée par l'administration de l'emploi durant cette première période ne semble pas obéir à la mise en œuvre d'une approche libérale explicite. Même si elle est portée par une " élite de l'emploi » depuis

8. «Le plus souvent, les membres de cette élite se situent dans les cabinets du Premier ministre et du ministre du Travail, dans les directions interministérielles ou dans des commissions ad hoc ou du Plan (délégation à 
la fin des années 1990, la progression d'une telle logique demeure largement motivée par des dimensions empiriques plutôt qu'idéologiques. Ces politiques visent en effet à répondre techniquement à l'accroissement de la charge pesant sur le SPE sans préjuger par principe une efficience plus grande du secteur privé. L'instrumentation particulière de l'action publique que représente le recours à des opérateurs externes publics comme privés se fait ainsi sans qu'une " conception concrète du rapport politique/société » et de sa régulation (Lascoumes, Le Galès, 2004) explicite ne puisse être mise en évidence. Les travaux du CGP concluent ainsi que "les pratiques de recours à des opérateurs externes n'ont pas été régulées par une doctrine préalable et explicite" (Balmary et al., 2004, p. 51). L'externalisation obéit à une rationalité binaire : une externalisation dite de "spécialité » dans les cas où sont soustraitées des prestations nécessitant une expertise dont l'opérateur public ne dispose pas en interne (c'était notamment le cas du conseil aux entreprises par exemple en matière d'ARTT) et de "capacité » lorsque l'administration de l'emploi externalise pour faire face à un accroissement de la demande à laquelle elle ne peut faire face sur la base de ses propres moyens humains. Mais c'est notamment sur ce second registre, le plus important numériquement, que la "neutralité instrumentale » mérite d'être questionnée. En effet, l'externalisation de capacité doit s'entendre au regard des choix politiques réalisés dans un champ de contraintes fortes. Le dilemme consistant à faire face à un chômage de masse cyclique tout en individualisant les prestations d'accompagnement et de placement - ce que Lavitry (2010) désigne sous le terme de "personnalisation de masse " - se comprend au regard de la volonté de ne pas accroître significativement le nombre d'agents statutaires de l'administration de l'emploi. Ce choix politique constant, quelle que soit la couleur partisane du gouvernement, est légitimé par un double discours articulant maîtrise des dépenses publiques et caractère cyclique du chômage. En cas de cycle de croissance du chômage, "l'externalisation de capacité " permet d'y faire face sans augmentation des effectifs de fonctionnaires pour les opérateurs publics (Berthet, 2010, p. 137).

\section{Une rupture et une phase d'institutionnalisation rapide (2005-2010)}

Un an après la parution du rapport faisant un premier état des lieux de l'externalisation, l'institutionnalisation de la sous-traitance connaît une brusque accélération. Ce tournant s'inscrit dans la mise en œuvre des préconisations européennes qui, de l'OCDE à la Commission européenne en passant par l'OIT, font valoir la nécessité d'ouvrir le marché du placement à des opérateurs privés (Vivès, 2013, 155sq.). L'OCDE invite expressément les États-membres à ouvrir les SPE à la concurrence depuis le début des années 1990. La convention $n^{\circ} 181$ de l'Organisation internationale du travail concernant les agences d'emploi privées (19 juin 1997) insiste sur le rôle positif des agences privées de placement dans le fonctionnement du marché du travail. Quant à la Stratégie européenne pour l'emploi (Conter, 2015), elle invite clairement les SPE nationaux à faire valoir leur plus-value spécifique par rapport aux opérateurs privés de placement. Ces préceptes, un temps freinés par l'importance symbolique du monopole de placement et la résistance de l'ANPE, vont être activés par les acteurs de l'indemnisation. C'est en effet le régime de l'assurance chômage qui

l'Emploi, direction du Budget ou de la Prévision) et de manière plus ponctuelle parmi les députés » (Colomb, 2012, p. 13). 
enfonce le premier coin par ses expérimentations et le gouvernement Raffarin qui ouvre la voie de l'institutionnalisation d'un marché concurrentiel du placement.

C'est par un texte législatif que s'ouvre cette phase d'institutionnalisation, la loi de cohésion sociale ${ }^{9}$ adoptée en janvier 2005 sous l'impulsion de Jean-Louis Borloo, ministre de l'Emploi, du travail et de la cohésion sociale du gouvernement Raffarin III. Cette loi définit l'activité de placement ${ }^{10}$ et, via une redéfinition du Service public de l'emploi, met un coup d'arrêt au monopole historique de l'ANPE en ouvrant la porte à une activité privée de placement ${ }^{11}$ et à la mise en concurrence de l'opérateur public (Soldini, 2006). Le levier déclencheur du changement de paradigme qui s'opère à partir de la loi de cohésion sociale est d'abord de nature cognitive. En effet, la notion de service public de l'emploi est redéfinie par le premier article de la loi de cohésion sociale. On passe d'une définition structurelle du SPE - c'est-à-dire par les organismes qui le composent - à une définition fonctionnelle basée sur les activités conduites auprès des demandeurs d'emploi ${ }^{12}$. Ce basculement cognitif est important parce qu'en faisant sauter le veto structurel, le gouvernement légitime la possibilité d'une modification organisationnelle majeure : l'ouverture du SPE à une catégorie élargie d'acteurs incluant des opérateurs privés de placement. Comme le rappelle Balmary : "Ainsi tout organisme, public ou privé, dont l'objet, ou l'un des objets, est d'intervenir sur le marché du travail, a vocation à "participer" à ce SPE » (Balmary, 2006). L'élargissement au champ et aux acteurs de l'indemnisation ouvre également la porte à de fortes oppositions quant au développement d'un marché de l'accompagnement et du placement au sein même du premier cercle du SPE (Béraud, Eydoux, 2009, p. 16). Comme le démontre Vivès dans sa thèse (2013), les oppositions mettent aux prises l'ANPE et l'Unédic tandis que les services centraux du ministère du Travail balancent d'une distance prudente et à une médiation timide entre ces deux acteurs fortement charpentés (voir également Ferracci, 2007). Ces tensions vont dans un premier temps se cristalliser autour d'une série d'expérimentations.

Comme il est de plus en plus souvent de rigueur dans l'introduction du changement en matière d'action publique, l'expérimentation tient également une place centrale (Chevallier, 2005) en amont de l'institutionnalisation. Le développement d'un marché privé du placement n'échappe pas à ce nouveau mode opératoire. Avant de revenir plus précisément sur cette phase expérimentale il convient toutefois de préciser rapidement de quel changement il est

9. Loi $n^{\circ}$ 2005-32 du 18 janvier 2005 de programmation pour la cohésion sociale.

10. «Art. L. 310-1. L'activité de placement consiste à fournir, à titre habituel, des services visant à rapprocher offres et demandes d'emploi, sans que la personne physique ou morale assurant cette activité ne devienne partie aux relations de travail susceptibles d'en découler. "

11. "Le service public de l'emploi comprend le placement, l'indemnisation, l'insertion, la formation et l'accompagnement des demandeurs d'emploi. II est assuré par les services de l'État chargés de l'emploi, l'Agence nationale pour l'emploi et l'Association nationale pour la formation professionnelle des adultes. II est également assuré par les organismes de l'assurance chômage mentionnés à l'article L. 351-21 dans le cadre des dispositions législatives et réglementaires qui leur sont propres. " "Les collectivités territoriales et leurs groupements concourent au service public de l'emploi dans les conditions prévues aux articles L. 311-9 et suivants. " "Peuvent également participer au service public de l'emploi les organismes publics ou privés dont l'objet consiste en la fourniture de services relatifs au placement, à l'insertion, à la formation et à l'accompagnement des demandeurs d'emploi, les organismes liés à l'État par une convention prévue à l'article L. 322-4-16, les entreprises de travail temporaire ainsi que les agences de placement privées mentionnées à l'article L. 312-1. »

12. "Le service public de l'emploi comprend le placement, l'indemnisation, l'insertion, la formation et l'accompagnement des demandeurs d'emploi. »Ibid., article 1. 
ici question. Comme nous venons de le voir, l'externalisation est une pratique ancienne, bien antérieure à la rupture qu'entraîne la fin du monopole de l'ANPE, et très développée au sein du SPE. Ce qui change n'est ainsi pas la nature de l'externalisation mais son étendue. En effet, avant son institutionnalisation en 2006, l'externalisation concerne des prestations que le SPE confie à des prestataires parce qu'il ne peut (externalisation de capacité) ou ne sait (externalisation de spécialité) pas les assumer. Mais c'est l'opérateur public qui maîtrise le chaînage des prestations et le parcours de placement du demandeur d'emploi. À partir de là, ce ne sont plus des interventions ponctuelles sur ce parcours qui sont externalisées mais l'intégralité des prestations d'accompagnement du parcours lui même. C'est en ce sens qu'il est convenu de parler d'opérateurs privés de placement (OPP). Deux questions émergent dès lors : comment se déroule le processus de développement d'un marché du placement ? Comment peut-on qualifier ce marché et sa régulation?

C'est sous une forme expérimentale et dérogatoire que l'ouverture de l'accompagnement et du placement se développe en premier lieu. Une institution tient ici un rôle-clé qu'il convient de rappeler, c'est le régime d'assurance chômage et ses opérateurs. La toute première brèche est ouverte dès 2003 par une agence Assédic de l'ouest francilien qui finance des prestations de placement proposées par la société néerlandaise Maatwerk dans le cadre des dépenses formation du PARE (Vivès, 2013, 225sq.). Bien que concernant un effectif très réduit de prestations (150), elle fait pourtant l'objet d'une contre-expérimentation (Objectif entreprise) de l'ANPE. À partir de janvier 2005, une seconde expérimentation est conduite par l'assurance chômage, en associant l'ANPE cette fois. Elle implique la société australienne INGEUS et concerne un effectif de 6000 demandeurs d'emploi. Là encore cette expérimentation est dérogatoire au droit commun et fait l'objet de tensions entre l'Unédic et l'ANPE notamment pour ce qui concerne son évaluation (Vivès, 2017).

Institutionnalisée par la loi de cohésion sociale et prévue par la convention tripartite État-ANPE-Unedic du 5 mai 2006, la mise en concurrence de l'opérateur public a été mise en œuvre dans les plus brefs délais puisque dès juillet de la même année, l'organisme d'indemnisation expérimente le placement de 41000 demandeurs d'emploi indemnisés par des OPP dans une dizaine de régions. C'est ainsi du cœur du SPE que provient cette initiative que l'ANPE tentera de contrer par une contreproposition de placement de 40000 demandeurs d'emploi dans six régions. Les deux opérateurs historiques du placement et de l'indemnisation se livrent ainsi bataille par expérimentations/évaluations interposées ${ }^{13}$. Les résultats autant que la méthode randomisée retenue sont peu probants et démontrent plutôt une meilleure efficacité du placement par l'ANPE ${ }^{14}$.

13. On notera de manière incidente que l'évaluation de ces expérimentations a été également l'un des fers de lance des tentatives de développer des méthodes d'évaluation expérimentales par assignation aléatoire. Comme le rappelle Devaux-Spatarakis, il est intéressant de noter ici à titre anecdotique que, bien que visant la régulation du marché du placement dans le cadre du code des marchés publics, le recours à ces évaluations " ne s'effectue pas dans le cadre de marchés publics mais de subventions de projets scientifiques avec le CREST et l'École d'économie de Paris » (Devaux-Spatarakis, 2014, p. 259).

14. Pour un bilan de ces expérimentations et de leurs évaluations [http://travail-emploi.gouv.fr/lMG/pdf/ Evaluation_Anpe_Unedic_au_02_02_10_pour_internet.pdf] (consulté le 23 octobre 2015). 


\section{9-2015: un marché qui se développe faiblement et un nouvel opérateur public qui renforce sa position}

La fusion des deux opérateurs au sein de Pôle emploi en 2009 rebat les cartes d'un point de vue organisationnel mais pas immédiatement au regard du développement d'un marché privé de l'accompagnement et du placement. Si la naissance de la nouvelle entité issue de la fusion de l'ANPE et de l'Unédic met fin aux concurrences entre opérateurs au sein du SPE, la mise en marché de l'accompagnement et du placement des demandeurs d'emploi se poursuit. Le contexte de développement de ce marché est important à prendre en compte. D'une part, et à la différence de la période précédente, il est marqué par un contexte de croissance forte du chômage engendré par la crise de 2008. Dans une logique d'externalisation de capacité, ce contexte d'explosion du nombre des demandeurs d'emploi constitue un moteur de développement de la sous-traitance.

\section{Graphique 1. Demandeurs d'emploi inscrits en fin de mois à Pôle emploi en catégorie $A$ au mois de janvier}

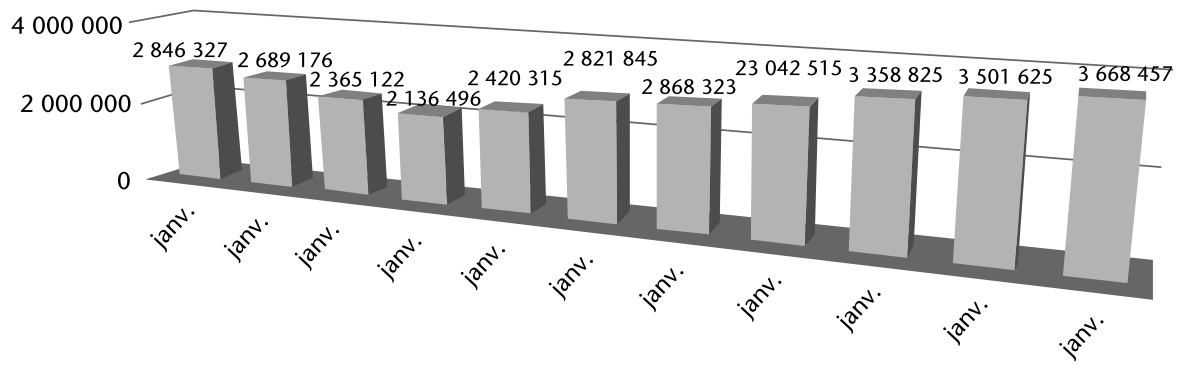

Source : Pôle emploi, STMT.

La réponse en termes de dépenses publiques dans les politiques d'emploi et notamment en termes de dépenses ciblées suit de près l'augmentation du chômage ${ }^{15}$.

\section{Graphique 2. Dépenses ciblées en faveur de l'emploi et du marché du travail en millions d'euros}

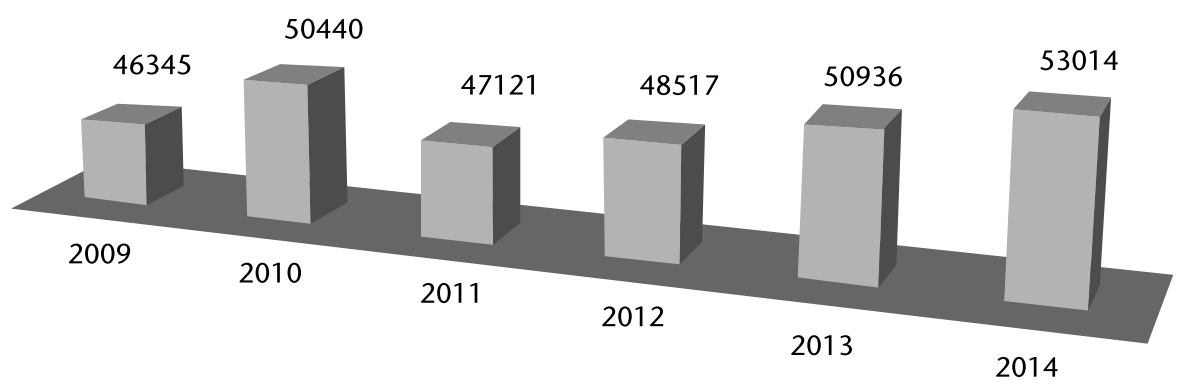

Source : DARES, PMT.

15. Les dépenses ciblées couvrent les « interventions publiques sur le marché du travail visant à permettre un fonctionnement efficace de celui-ci et à corriger des déséquilibres, et qui peuvent être distinguées d'autres interventions plus générales de la politique de l'emploi dans la mesure où elles agissent de façon sélective en favorisant des groupes particuliers sur le marché du travail » selon la définition retenue par Eurostat. 
En termes de recours à des OPP, à partir de septembre 2009, deux prestations sont mises en œuvre. La première - Trajectoire vers l'emploi (TRA) - vise l'accompagnement renforcé de personnes présentant un risque élevé de basculement dans le chômage de longue durée. Le second dispositif - Licenciés économiques (LEC) - s'adresse à des salariés en transition professionnelle. Deux ans plus tard, 206550 demandeurs d'emploi ont été pris en charge dans le cadre du premier dispositif et 1190980 dans le cadre du second (Pôle emploi, 2012). 31 prestataires pour 64 lots ont été retenus dans le cadre de la procédure d'appel d'offre (Cour des comptes, 2014). En juin 2010, deux nouvelles prestations font l'objet d'une procédure de marché (Atouts cadres et Mobilisation vers l'emploi ${ }^{16}$ ), puis en 2012 une nouvelle vague de prestations est proposée aux OPP ${ }^{17}$.

II ne faudrait pas en conclure pour autant à la croissance inéluctable d'un marché privé du placement qui s'accompagnerait d'un affaiblissement de l'opérateur public, Pôle emploi. Le bilan détaillé réalisé par la Cour des comptes en mai 2014 démontre exactement le contraire. Le volume de prestations externalisées ne cesse de se réduire en dépit de la croissance constante du nombre des demandeurs d'emploi sur la période concernée. Après une période de forte croissance entre 2009 et 2010, le recours à la sous-traitance baisse de manière continue à partir de 2011. Toutes prestations externalisées confondues, «le nombre de prescriptions a diminué de moitié (53,5 \%) entre 2009 et 2013 »(Cour des comptes, 2014, p. 31).

\section{Graphique 3. Nombre de prestations d'accompagnement sous-traitées par Pôle emploi}

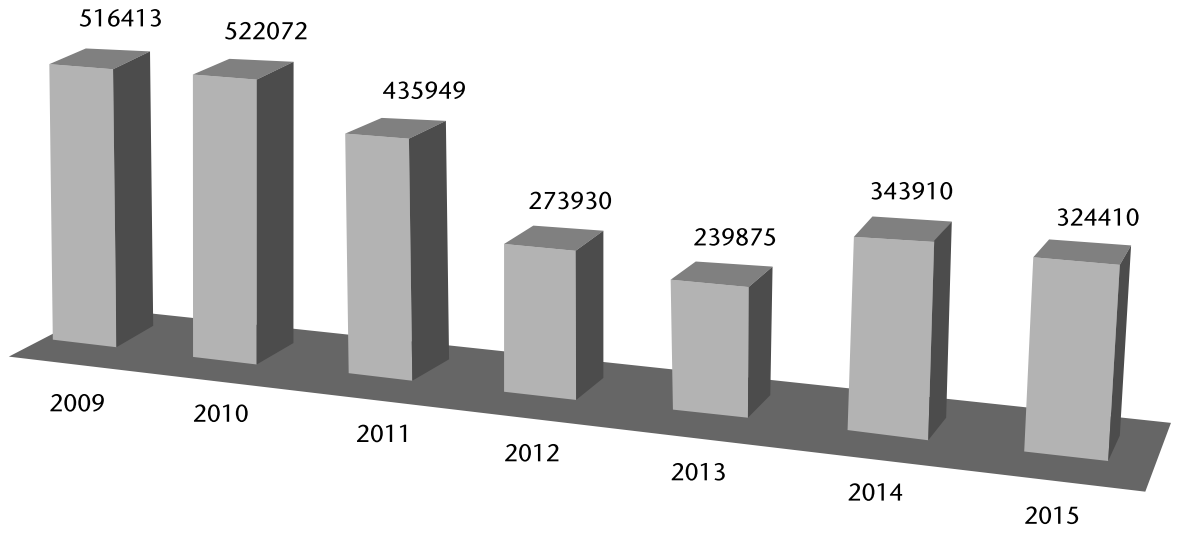

Sources : Cour des comptes (2009-2013) et rapports d'activités Pôle emploi (2014-2015).

Au final, le recours aux OPP demeure un phénomène encore minoritaire y compris en volume financier. II représente, en 2013, 2,9 \% des dépenses de Pôle emploi soit 145 millions d'euros au sein d'un budget d'intervention et de fonctionnement avoisinant les 5 milliards d'euros (Assemblée nationale, 2014, p. 16). Les motifs de cette faible adhérence sont

16. Le premier dispositif vise comme son nom l'indique un public de cadres tandis que le second s'adresse à un public présentant des difficultés personnelles et sociales.

17. Cela concerne des prestations courtes de positionnement tous publics ; un dispositif pour jeunes diplômés (Du diplôme à l'emploi), pour des chômeurs peu éloignés de l'emploi (Objectif emploi) et pour des demandeurs d'emploi de longue durée (Trajectoire vers l'emploi). 
multiples. Ils sont liés au souci d'un retour à l'équilibre financier pour l'opérateur public et à des choix locaux de privilégier les prestations réalisées en interne. Ils tiennent également aux conditions d'organisation et de régulation de ce marché qui brident sa rentabilité pour les OPP (Cour des comptes, 2014). Le faible développement d'un marché privé du placement se traduit également en termes d'effectifs chez Pôle emploi. Loin d'aboutir à un affaiblissement de l'opérateur public, il s'accompagne d'un renforcement de ses effectifs.

\section{Graphique 4. Effectifs de Pôle emploi en ETPT}

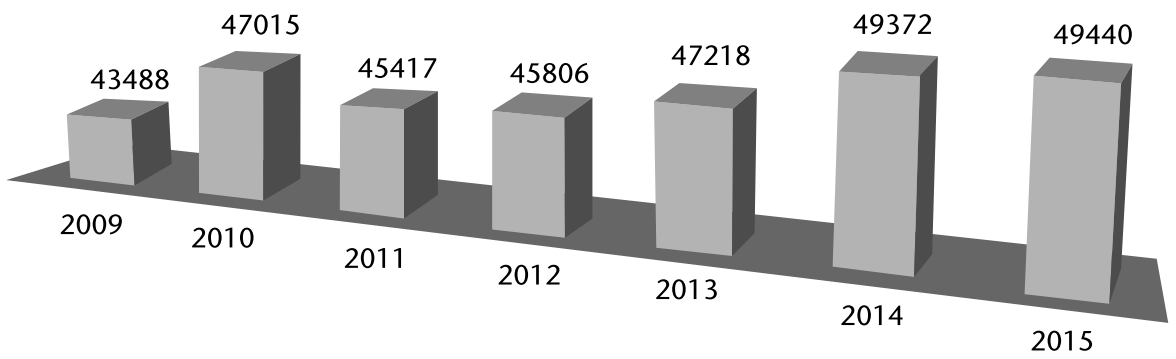

Source : Cour des comptes et Pôle emploi.

Entre 2011 et 2014, Pôle emploi embauche ainsi 3955 ETPT principalement en CDI (94\%) compensés dans son budget par une baisse des achats et services extérieurs (-90 millions d'euros), des prestations externalisées (- 95 millions d'euros) et des dépenses ( -100 millions d'euros) liées à la sous-traitance (Cour des comptes, 2015, p. 38).

Ce qui n'empêche pas la France de poursuivre l'institutionnalisation de cette pratique en ratifiant, après douze autres pays membres de l'Union européenne, au printemps 2015 la convention $\mathrm{n}^{\circ} 181$ de l'Organisation internationale du travail encadrant le recours à des opérateurs privés de placement sous condition de gratuité pour les demandeurs d'emploi et de non-discrimination. La loi $n^{\circ}$ 2015-278 du 13 mars 2015 qui autorise cette ratification énonce pour objectif d'accroître « les capacités globales d'accueil et de prise en charge des demandeurs d'emploi, et d'autre part d'accroître la collecte et la diffusion d'offres d'emploi vacants ainsi que la qualité des réponses susceptibles d'être apportées à celles-ci, tout en conservant au service public de placement son rôle pivot ». Concernant ce dernier point, la signature de la convention 181 - dix-huit ans après sa publication par l'OIT - demeure strictement encadrée par une logique de sous-traitance où le service public de l'emploi demeure commanditaire. Deux raisons sont évoquées par l'étude d'impact de la loi du 13 mars : " D'une part, les services de Pôle emploi étant gratuits pour les entreprises, celles-ci n'ont pas un intérêt économique à avoir recours directement aux agences d'emploi privées dont les services sont payants ; d'autre part, le marché du placement n'est pas encore très développé en raison de l'ouverture relativement récente aux agences d'emploi privées. " (ministère des Affaires étrangères et du développement international, 2015.)

\section{Comment qualifier l'institutionnalisation de ce marché et sa régulation?}

Le marché du placement se caractérise ainsi en France par sa dimension encore fortement régulée. La marchandisation comme instrumentation de l'action publique reposant sur 
le recours aux marchés publics engage une série de dimensions analytiques. Ces dimensions, formalisées par Smith et Ansaloni dans la foulée des travaux sur les institutions des marchés (Polanyi, 2011 ; Tordjman, 2004) en introduction à ce numéro spécial, permettent d'opérationnaliser les notions larges et floues de marché et de marchandisation. Ainsi, l'analyse des différentes composantes institutionnelles de ce marché - identité des participants, attributs de la marchandise échangée, organisation des transactions et valeur d'échange - dans une perspective diachronique, permet de mettre en évidence une tendance au développement d'une régulation concurrentielle subventionnée dans un cadre fortement administré.

Du côté des participants, une transformation des acteurs du marché s'opère progressivement. Une première génération ${ }^{18}$, souvent désignée sous l'appellation générique d'« intermédiaires » du marché du travail ou de l'emploi (Bessy, Eymard-Duvernet, 1997 ; Gelot et al., 2000), s'est structurée dans la logique d'externalisation séquentielle. Ce premier marché est composé d'organismes de formation privés ou publics, d'associations du secteur de l'insertion et de cabinets de conseil. À cette première génération s'ajoute, à partir de l'ouverture du marché du placement introduit par la loi de cohésion sociale de 2005, une seconde catégorie d'opérateurs (Divay, 2009, p. 40) généralement identifiés comme "opérateurs privés de placement"(OPP). Parmi ces opérateurs on retrouve des agences d'intérim, des cabinets de ressources humaines, des entreprises d'insertion, des entreprises de travail temporaire et surtout des agences privées de placement rattachées ou pas à des grands groupes internationaux. Comme le rappellent Béraud et Eydoux (2009), ces OPP se sont parfois stratégiquement investis sur la première génération d'externalisation pour mieux se positionner ensuite sur le marché des prestations intégrées ${ }^{19}$.

Les attributs de la marchandise ont évolué et sont au cœur du passage de l'externalisation séquentielle à la marchandisation globalisante du placement. Tout en demeurant par nature dans le champ de la délivrance d'un service, l'offre publique mise en marché a évolué dans sa structure. On est ainsi passé de l'achat de prestations ponctuelles dans le cadre d'une maîtrise d'œuvre publique à l'achat de prestations intégrées d'accompagnement et de placement évaluées sur les résultats en termes de situation individuelle des bénéficiaires. Bien que juridiquement non assimilable à une délégation de service public, ce changement étend considérablement la nature du service mis en marché.

Le mode d'organisation des transactions reste encadré par le code des marchés publics et la logique de sous-traitance qui l'accompagne. Distinguant en cela l'achat de prestations de la délégation de service public, le régime du code des marchés public demeure un verrou cohérent au regard des logiques d'action. En effet, la sous-traitance à des OPP pour des motifs de capacité ou de spécialité suppose que ce recours soit conduit sous maîtrise d'œuvre de l'administration de l'emploi et dans un but de complémentarité à son action propre $^{20}$. Le service public du placement et de l'accompagnement n'est ainsi pas délégué

18. Nous empruntons ici la terminologie de "générations » à Divay (2009).

19. Ils évoquent notamment le cas de l'entreprise Adecco (un des principaux opérateurs avec Altédia et BPI) qui, dans un premier temps, a diversifié ses activités de travail temporaire en se positionnant sur des prestations externalisées par le SPE pour ensuite développer une activité plus intégrée d'OPP.

20. En ce qu'elle supposerait la gestion contractualisée d'un service public par le délégataire ce qui est antagonique à la mise en marché public pour répondre aux besoins des opérateurs publics « en matière de 
mais " marchandisé » dans un cadre où l'opérateur public demeure central, voire renforce sa «position hégémonique » (Vivès, 2014) par les conditions drastiques (cadrage de la prestation, prix, modalités de suivi) qu'il impose à ses prestataires.

Dans ce cadre de marché administré, la valeur d'échange de la marchandise - son prix - est très précisément contrôlée par les autorités publiques. Élément central de la marchandisation (Vatan, 2013), le prix des prestations mises en marché par le SPE est strictement encadré. C'est également sur cette base que s'est principalement effectuée la sélection des opérateurs par Pôle emploi et ce au détriment des attributs techniques de la marchandise. En effet, alors que l'appréciation de l'offre technique des OPP constituait un critère dominant au plan formel dans le choix des prestataires, le cadrage extrêmement rigoureux du contenu des prestations a abouti dans les faits à faire de l'offre tarifaire le principal critère discriminant (Cour des comptes, 2014, p. 44). Cet état de fait a eu pour conséquence de conduire les OPP à une course délétère au moins disant financier ${ }^{21}$.

Au final, le marché du placement et de l'accompagnement des demandeurs d'emploi demeure placé sous une étroite régulation par le SPE et soumis à une logique de soustraitance administrée. Bien qu'en constante progression depuis le milieu des années 2000, la marchandisation du service public de l'emploi - rendue possible par une définition fonctionnelle de celui-ci - connaît, depuis 2011, un recul quantitatif qui replace l'opérateur public Pôle emploi au centre de sa régulation et aboutit à utiliser " désormais les prestataires privés comme variable d'ajustement » (Vivès, 2014, p. 4). C'est sur cette base qu'une réorientation a été décidée par son conseil d'administration en février 2014. Elle inscrit désormais la stratégie de recours à des prestataires dans un partage des tâches où l'opérateur public réinternalise les actions en faveur des demandeurs d'emploi les plus éloignés du marché du travail (environ 137000 personnes) et externalise davantage l'accompagnement des quelques 500000 demandeurs d'emploi les plus autonomes (Assemblée nationale, 2014, p. 12).

\section{Les parcours des demandeurs d'emploi face à la marchandisation : un équilibre asymétrique entre opérateurs}

La genèse de la sous-traitance dans le champ de l'emploi éclaire ainsi les changements de modes de gouvernance, la place de l'État et le rôle des marchés. Mais ces changements de cadre normatif pour l'action publique ont également des conséquences sur la mise en œuvre concrète des dispositifs de la politique de l'emploi et sur les pratiques des conseillers

travaux, de fournitures ou de service " au sens des définitions donnés par la loi Sapin n $93-122$ du 29 janvier 1993.

21. La Cour des comptes note ainsi dans son rapport relatif au recours aux OPP par Pôle emploi que : La stratégie de conservation ou de conquête de parts de marché a ainsi pu conduire certains prestataires à négliger la réalité économique ou à étayer leurs propositions tarifaires par des hypothèses trop optimistes sur la reprise économique et par conséquent sur leurs performances en termes de reclassement. D'autres, au contraire, jugeant la baisse des prix excessive, ont préféré ne pas se porter candidat lors du renouvellement des marchés ou concentrer leur activité sur des segments jugés moins concurrentiels (Cour des comptes, 2014, p. 51). 
en insertion professionnelle. En déplaçant l'analyse sur ce niveau opérationnel, on peut mettre en évidence les décalages entre la décision politique et les conditions de sa mise en œuvre effective (Pressman, Wildavsky, 1984 [1973]). Ce décentrement du regard permet de mettre en discussion, "par le bas ", la manière dont les logiques de marchandisation de l'accompagnement des demandeurs d'emplois rencontrent les représentations et pratiques des opérateurs de terrain.

Au moment de l'enquête de terrain qui informe cette section (2011-2014), le basculement entre l'achat de prestations ponctuelles vers l'achat de prestations intégrées d'accompagnement de parcours est engagé. Pour autant, la mise en marché de prestations globales s'inscrit dans un cadre d'externalisation déjà routinisé et n'apparaît pas comme un point de rupture. Les références régulières à une sorte d'âge d'or aujourd'hui révolu des pratiques d'accompagnement englobe un ensemble de changements organisationnels (fusion ANPE/Pôle emploi) et dans les pratiques de management qui exprime avant tout un sentiment de perte d'autonomie des agents auquel participe la mise en marché ponctuelle comme globale de l'accompagnement des demandeurs d'emploi. Ce changement est vécu sous le mode de l'approfondissement d'une dynamique institutionnalisée. Le recours à des opérateurs externes est une pratique ancienne d'accompagnement de catégories de publics cibles comme par exemple, les jeunes ou les demandeurs d'emploi de longue durée (Parent et al., 2014). Les conseillers de l'ANPE puis de Pôle emploi, orientent ainsi les jeunes vers les missions locales, suivant une logique de spécialisation des organismes et de cotraitance de l'accompagnement des parcours individuels. Face au développement de l'individualisation et d'une approche globale de l'individu comme paradigme d'accompagnement (Van Berkel, Valkenburg, 2004), cette tendance à l'externalisation des accompagnements de certains publics-cible s'est renforcée (Georges, 2007) jusque dans les années 2010. Les missions locales, les PLIE (plans locaux pluriannuels pour l'insertion et l'emploi) et d'autres organismes privés spécialisés (du secteur marchand ou non marchand) accompagnent les demandeurs d'emploi qui leur sont prescrits par Pôle emploi ou d'autres prescripteurs (comme les conseils départementaux par exemple qui ont notamment recours à des prestataires privés pour accompagner les bénéficiaires du RSA).

Cette section vise à montrer que l'introduction de mécanismes de marché a fait néanmoins évoluer la nature des relations qu'entretiennent les acteurs en charge de mettre en place le service externalisé avec le prescripteur. Ainsi, alors que les acteurs privés œuvrant dans le secteur de l'emploi ont, au cours de la première phase d'externalisation séquentielle analysée dans cet article, travaillé en collaboration avec Pôle emploi dans une logique de partenariat visant la construction de parcours d'insertion professionnelle, ils ont ensuite été progressivement positionnés comme de simples prestataires de services. L'analyse des échanges entre les parties prenantes à cette relation de service permet de mettre en lumière le renforcement des rapports de force entre commanditaire et prestataires ainsi que les conséquences de la marchandisation des accompagnements vers l'emploi sur les pratiques professionnelles des agents. Ce passage d'une relation de partenaire à celle de prestataire, régulièrement évoquée par les agents interrogés, s'incarne dans la nature des instruments mis en place pour réguler l'intervention des opérateurs, leur mise en œuvre et les relations qu'ils installent entre les protagonistes de cette relation marchandisée. 
Nos observations sur les effets de la mise en marché de l'accompagnement des demandeurs d'emploi ont été conduites dans trois unités urbaines françaises (Bordeaux, Tours et Montpellier) présentant des traits de contraste et de comparabilité.

Un premier constat s'impose au regard de la différenciation locale : l'enquête comparée réalisée sur trois unités urbaines ne permet pas de mettre en évidence une variation territoriale significative au regard des pratiques des opérateurs. Malgré des situations contrastées, les phénomènes décrits dans cette section sont ainsi transversaux à ces trois métropoles régionales.

L'analyse approfondie des 87 entretiens réalisés sur ces trois sites met en évidence la prééminence de trois principaux niveaux de changements :

- la nature des échanges entre donneurs d'ordre et prestataires et la marge de manœuvre dont ces premiers disposent ;

- la manière dont la marchandisation conduit à une logique de volumétrie contraire à la spécialisation de l'accompagnement sur des publics cibles;

- enfin, les stratégies que déploient les agents de terrain pour contourner ces logiques de mise en marché.

Cette seconde section est centrée sur l'analyse de ces trois dynamiques de changement qui convergent pour souligner les dysfonctionnements structurels (Dubois, 2013) et le rôle de ces acteurs de terrain (Lipsky, 1980 ; Rice, 2015) dans la mise en œuvre concrète de l'externalisation.

\section{Une marge de manœuvre contrainte par le marché}

Dans les années 2010, en pleine période de croissance de la sous-traitance globale, la direction nationale de Pôle emploi insiste également sur l'importance de donner davantage de marge de manœuvre aux conseillers en insertion professionnelle (Pôle emploi, 2012). Pour autant, la logique de mise en marché contraint cette autonomie en introduisant une relation financière qui repose avant tout sur une relation de donneur d'ordre à prestataire (Vivès, 2013; Howe, 1991). Signalés de manière régulière lors des entretiens réalisés avec des opérateurs de première ligne, les changements organisationnels à Pôle emploi sont vécus comme porteurs de contraintes à l'exercice de l'accompagnement des demandeurs d'emploi : "Il y a une autonomie qui a été perdue, après la fusion, par rapport à ça. Certainement, c'est-à-dire qu'on était beaucoup plus libres dans le cadre ANPE que dans le cadre Pôle emploi » (conseiller, agence Pôle emploi).

Les modes de coopération entre acteurs et organisations dans le secteur de l'emploi ont évolué au cours des deux dernières décennies en renforçant le caractère formel et la rigidification des relations. Ainsi que le rappelle ce directeur régional, c'est bien au plus près du terrain que se concentrent les contraintes : "Nous on est dans un système emboîté dans lequel chaque niveau est obligé de jouer et le dernier niveau local qui devrait être celui où on a le plus de mobilité d'action, est le niveau où convergent toutes les modalités de contraintes ! C'est le paradoxe. Ça n'empêche pas d'agir mais ça n'aide pas. "Les relations entre agents de Pôle emploi et opérateurs externes s'organisent à présent autour de rapports de force qui réinterrogent leurs modalités de travail. Face à une diminution de liberté d'action 
quant au choix du prestataire et à une rigidification de leurs rapports directement induite par les instruments de pilotage de cette mise en marché ; les relations entre opérateurs des politiques de l'emploi connaissent des changements de nature et de modalités de coopération. "Ce n'est pas le même rapport. On, je, on est financeurs, donc on est donneur d'ordre » (conseillère, agence Pôle emploi). Deux éléments-clés sont régulièrement mis en évidence par nos interlocuteurs et permettent de les éclairer : la manière dont le prestataire de l'accompagnement du demandeur d'emploi est choisi et la nature des échanges entre donneurs d'ordre et prestataires.

Avant l'institutionnalisation de la sous-traitance globale (voir supra), les conseillers pouvaient choisir l'organisme vers lequel orienter le demandeur d'emploi en fonction de leur connaissance des prestataires locaux. Ce responsable d'agence Pôle emploi l'exprime clairement lors de notre entretien : "Mais c'est surtout qu'on s'appuyait sur des collaborations. Moi je vois à X. qui était une ville avec beaucoup d'organismes, il y avait du travail pour tout le monde. Aucun organisme qui pouvait se plaindre d'avoir moins de commandes que les autres. II y avait quatre agences, pas de favoritisme particulier et on savait qu'à untel il fallait lui envoyer les gens qui avaient des difficultés avec l'écrit et l'oral et il ferait des miracle avec, untel on savait sur les cadres parce qu'il avait un gros réseau d'entreprises et il ferait du super boulot, et puis vous aviez des relations directes entre le conseiller et le prestataire. Moi je ne m'en mêlais pas, c'était mes conseillers, ils avaient un gars en face d'eux, ils appelaient le prestataire en disant: "Voilà, monsieur untel ou madame est-ce que tu peux prendre en charge ?" Et le prestataire renvoyait au conseiller les informations en retour. On arrivait à une qualité de prise en charge et qui était moins perçue par les gens parce qu'ils savaient qu'ils allaient dans le cabinet du coin. Aujourd'hui, ils ont l'impression qu'on les balade chez des opérateurs privés, des grosses boutiques. C'est le paradoxe! On essaie de les envoyer pour qu'ils aient une prestation plus individualisée, et en fait ils se retrouvent pris dans des trucs qui sont aussi avec des règles de productions industrielles. " Le choix reposait alors sur la spécialité de l'organisme (sur un type d'accompagnement ou un type de public). Ainsi, face à un demandeur d'emploi étranger connaissant des difficultés de reconnaissance de diplômes et de compréhension des codes culturels, le conseiller pouvait l'orienter vers l'acteur qu'il jugeait le plus à même de répondre aux besoins grâce à sa connaissance du tissu partenarial local. Cette logique de spécialisation persiste et c'est même l'un des principaux arguments justifiant le recours à la sous-traitance de l'accompagnement, mais le processus de sélection du prestataire change avec la loi de cohésion sociale (Vivès, 2013). Désormais, les conseillers de Pôle emploi ne choisissent plus le prestataire qu'ils jugent le plus à même de répondre aux besoins du demandeur d'emploi. " Le choix des prestataires, ce n'est pas de notre ressort. II y a des appels d'offre et c'est la direction qui choisit. Je sais qu'il y a eu des castings qui ne nous semblaient pas bons... » (conseillère, agence Pôle emploi). Ils sont, dans la plupart des cas, contraints de sélectionner le prestataire qui a remporté un appel d'offre, ce qui est perçu par les agents, qu'ils soient opérateurs de première ligne ou managers, comme un facteur de contraintes et non comme une manière de structurer le marché des prestations. Un responsable au sein d'une direction régionale de Pôle emploi explique ainsi que: "C'est vrai que nous, au niveau local, on préférait travailler avec des petites boutiques parce que vous l'appelez le matin pour lui dire: "à 10 heures, j'ai un gars, faut que tu le vois", et la petite boutique dit: "envoie-le moi." 
C'était des relations de vrais partenariats, des gens impliqués sur un bassin d'emploi. Et ça, franchement, je trouve qu'on a perdu énormément » (cadre, direction régionale de Pôle emploi).

Pour les conseillers, la logique de mise en concurrence instaurée par la marchandisation de l'accompagnement est synonyme de rigidification du processus de sélection des prestataires. La systématisation de cette logique limite par ailleurs la mise en place de partenariats en dehors du cadre réglementaire imposé. Ce que rappelle un responsable de service à Pôle emploi : "Les lois sur les marchés contraignent beaucoup plus. C'est-à-dire qu'on a connu, enfin l'ANPE connaissait plus une ligne expérimentale. Aujourd'hui, la direction générale voudrait la ré-ouvrir [...]. Mais c'est beaucoup plus compliqué à écrire aujourd'hui parce qu'il ne faut pas qu'on puisse être attaqué sur un détournement des marchés » (responsable de service, agence Pôle emploi).

Nos entretiens ont permis de faire ressortir l'importance des relations informelles existantes entre acteurs locaux dans l'accompagnement du demandeur d'emploi : le conseiller le met régulièrement en contact avec les autres acteurs de l'emploi qu'il connaît et avec lesquels il échange régulièrement. Ce constat va dans le sens des premiers travaux de la sociologie des organisations qui insistaient sur le poids de ces relations informelles dans le fonctionnement des organisations (Crozier, Friedberg, 1977). Pourtant, la logique des marchés publics ayant pour objectif la mise en place d'une régulation de la concurrence laisse peu de place aux relations informelles dans le choix du prestataire et dissout la proximité des acteurs. "On a aussi la notion de non-préférence concurrentielle, c'est-à-dire je ne vais pas pouvoir, même si je trouve une association qui offre un service, même s'il est pas payant, mais qui pourrait être concurrentiel avec un service payant, même si je trouve que c'est formidable, je vais pas pouvoir en faire la publicité auprès des demandeurs d'emploi, pour être clair, puisque je pourrais être attaqué enfin d'avoir mis en œuvre une concurrence déloyale » (conseillère, agence Pôle emploi). II existe deux raisons principales à cela : d'une part, l'impossibilité de choisir le prestataire en fonction de sa connaissance des acteurs locaux; d'autre part, la mise en place d'instruments pour échanger sur le parcours et l'accompagnement du demandeur d'emploi qui renforcent les rapports de force entre le donneur d'ordre et le prestataire dans la mesure où ils ne permettent pas une communication entre les différentes parties prenantes (Divay, 2009). Une conseillère Pôle emploi explique ainsi que «les prestataires les plus couramment utilisés, oui ils peuvent nous joindre. Voilà. Sur des dispositifs exceptionnels, ils ont au moins un correspondant à défaut d'avoir le collègue qui a prescrit lui-même ". Tandis que du côté des prestataires le manque de communication est régulièrement mentionné comme en témoigne cet extrait d'entretien avec un prestataire PLIE : «Pôle emploi, c'est plus difficile d'avoir accès à leurs conseillers de manière directe. Après, ils ont des outils pour nous renseigner, mais c'est parfois plus opaque. » La mise en œuvre de la sous-traitance ne passe donc plus par ces échanges informels, mais principalement par des fiches navettes qui permettent de faire passer un certain nombre d'informations du prescripteur au prestataire et vice versa. Cependant, ces documents ne permettent que rarement d'expliquer en détails ce qui a été réalisé et/ou détecté concernant l'accompagnement du demandeur d'emploi. Un conseiller explique ainsi que lorsqu'un prestataire a travaillé sur des problématiques d'addiction qui représentent un frein majeur à l'emploi, il n'a pas l'espace matériel pour le mentionner sur sa fiche de suivi. II en découle 
que si le demandeur d'emploi est ensuite amené à retourner vers le prescripteur, ce dernier ne disposera pas de cette information importante le concernant. En dépit du développement d'une externalisation globale, les procédures de partage d'informations entre les parties prenantes ne permettent donc pas de décrire l'accompagnement réalisé dans son intégralité. Ils sont centrés sur des critères quantitatifs ou ciblés sur l'emploi et ne prennent pas en considération les éléments périphériques traités par le prestataire. L'institutionnalisation de la logique de marchandisation participe ainsi à des changements de relations dans la mesure où elle instaure un rapport d'acheteur à prestataire. Dès lors, ces derniers se sentent l'objet d'un rôle purement instrumental.

\section{Une logique de volumétrie qui met à mal la spécialisation sur les publics}

Un deuxième effet de la mise en place d'une dynamique de marchandisation dans le secteur de l'accompagnement des demandeurs d'emploi est la logique de volumétrie qu'elle induit (Parent et al., 2014) et qui met à mal le processus d'individualisation. Le SPE a recours à des prestataires extérieurs pour des publics spécifiques au regard desquels des organismes sont spécialisés (les bénéficiaires du RSA par exemple). Néanmoins, face à la nécessité de se regrouper pour répondre aux marchés publics et face à une commande publique centrée sur certaines catégories de demandeurs d'emplois, les prestataires ont été amenés à se diversifier pour répondre à la commande et à mettre de côté leur spécialisation. La sous-traitance des accompagnements conduit les organismes prestataires à transformer leurs pratiques pour être en adéquation avec les marchés proposés en dépit de leurs spécialisations propres. "lls n'ont pas le choix par moment, et sinon ils meurent. Là on a un vrai sujet que je remonte aux politiques depuis des années et aujourd'hui la politique d'achat avec les contrats qu'on a lié au seuil de marché, qu'on considère le marché de la prestation, c'est Pôle emploi, c'est l'enveloppe nationale qu'on regarde pour définir le marché » (cadre, direction régionale Pôle emploi).

Pour répondre aux appels d'offre, bon nombre d'organisations de petite taille doivent ainsi se regrouper avec de plus gros organismes qui, eux, sont habitués à répondre à ce type de marchés et disposent des ressources nécessaires pour produire une réponse correspondant aux critères de sélection des prestataires. Cependant, cela se fait souvent au détriment des spécialités de chacun et dans une standardisation croissante de l'offre publique au moins pour les prestations ponctuelles comme le rappelle cette conseillère de Pôle emploi : "Oui, c'est standardisé [...] on fait beaucoup plus de standardisation sur des prestations qu'on gère, les prestations courtes sont standardisées. L'accompagnement c'est une prestation, entre guillemets, un suivi sur la longueur, ça peut pas être standardisé, c'est... même nous on tiendrait pas. " Ainsi, une conseillère d'une association spécialisée auprès des demandeurs d'emploi immigrés explique que la structure a dû se regrouper avec d'autres pour remporter un marché. Le mandataire du marché, c'est-à-dire celui qui est responsable du groupement, cherche à placer les demandeurs d'emplois qui correspondent aux spécialités des uns et des autres. Cependant, la conseillère explique : " Il y a des logiques de volumétrie que le mandataire prend en compte, c'est-à-dire qu'au bout d'un moment, si tu as trop de prescriptions, il va être obligé de dispatcher à quelqu'un d'autre pour respecter les engagements de chacun [...]. Le groupement a été fait de façon très mathématique 
dirons-nous [...]. Et puis une logique de spécificité puisqu'ils ont fait appel à nous connaissant notre spécificité mais avec des logiques de volumétrie qui, mine de rien, posaient problème car du coup, des prestataires du mandataire se retrouvaient avec des publics dont ils ne maîtrisaient pas forcément la méthodologie, l'approche, etc., et nous, à l'inverse, on se retrouvait pareil avec des publics par exemple des cadres, des ingénieurs, où on n'avait pas forcément les outils appropriés " (conseillère en insertion au sein d'un organisme privé). La volonté de sous-traiter les accompagnements ciblés à des prestataires extérieurs est donc freinée par les logiques induites qu'elle génère. Ces dernières rigidifient les relations partenariales entre acteurs : les organisations passant d'une "posture de coordination des interventions à un rôle de commanditaire " (Berthet, 2012), et les opérateurs d'une posture de partenaire à celle de prestataire.

Cette logique de volumétrie ne concerne pas seulement les prestataires, elle touche également les agences locales de Pôle emploi qui ne peuvent décliner des actions qu'à condition de pouvoir positionner un nombre minimum de demandeurs d'emploi. Cela conduit parfois à laisser de côté les plus petites communes : "Le cadre juridique est épouvantable. Aujourd'hui, c'est proche du carcan. On a maintenant des volumes obligatoires, ce qui peut être logique, de mise en œuvre de prestations qui fait que sur [notre commune] par exemple, il y a des prestations que je ne peux pas ouvrir, parce que je ne pourrais pas garantir, je n'ai pas assez de demandeurs » (conseiller, agence Pôle emploi). Les conseillers en insertion sont ainsi directement concernés eux aussi par les nouvelles conditions qu'impose la mise en œuvre des règles de marchés publics et ils adaptent leurs pratiques professionnelles à ce nouveau cadre, quitte à le contourner parfois.

\section{Des contournements de la logique de marché}

Les travaux de sociologie de l'action publique sur le rôle des " street level bureaucrats » (ici les conseillers en insertion) soulignent l'importance de l'évolution de la marge de manœuvre dont disposent ces acteurs en fonction de l'évolution des systèmes managériaux dans lesquels ils évoluent. Howe (1991), critiquant l'analyse de Lipsky (1980) qui attribuait un fort pouvoir discrétionnaire aux agents de terrain, estimait que le développement d'un système proche du New Public Management conduirait à la mort de la discrétion des agents de terrain. Or, les entretiens auprès des conseillers en insertion ont permis de constater que si les conseillers perçoivent la marchandisation de l'accompagnement comme une contrainte et un système rigide ce que rappelle ce conseiller : " certainement, c'est-à-dire qu'on était beaucoup plus libres dans le cadre ANPE que dans le cadre Pôle emploi » (conseiller, agence Pôle emploi) ; ils peuvent néanmoins se ménager des marges de manœuvre dans le champ de l'accompagnement. " Autant sur l'indemnisation il n'y a pas de marge de manœuvre, la personne elle a des droits ou elle en a pas, autant sur la détermination de l'accompagnement, le conseiller a une marge de manœuvre " (conseillère, agence Pôle emploi). Si l'offre de service est construite au niveau national, comme le rappelle cette conseillère indiquant en entretien qu'« en tant que Pôle emploi, je n'ai pas, aujourd'hui, de possibilité d'initier un dispositif inexistant. Je vais utiliser des outils existants, ils vont avoir plus d'importance ici que... en fonction de l'analyse du territoire et de la part de la bonne volonté des acteurs locaux, mais je vais rester dans le cadre, enfin je... je suis un établissement national, je vais rester dans le cadre d'un établissement national "; elle peut néanmoins à l'adapter aux 
besoins de son territoire : " Je vais disposer, sous une forme ou sous une autre, d'un budget d'intervention au titre de la formation. Je vais pouvoir déterminer quel type de formation je souhaite qui soit financée pour l'année prochaine, sur mon département. Par contre, je ne peux pas dire avec cet argent pour la formation, je vais faire autre chose. Voilà, je l'utiliserai à l'objet. Je l'utiliserai à la formation. Par contre, en fonction de l'analyse de... des besoins en main-d'œuvre sur mon territoire, je vais pouvoir infléchir, dire je souhaite plutôt des formations cariste, je souhaite plutôt des formations de, enfin sur, dans une enveloppe contrainte je vais pouvoir modifier non pas l'objet générique, ça sera de la formation mais je vais pouvoir rentrer dans le détail et infléchir sur le, enfin, dans l'exemple, le type de formation que je vais... je vais mettre en œuvre ". En s'appuyant sur la marge de manœuvre dont ils disposent et en élaborant des stratégies basées sur leur connaissance du système et des territoires, certains adaptent le cadre afin de répondre à leurs besoins et à leur méthode de travail. "On a une marge de manœuvre sur l'accompagnement qu'on met en place, par contre, on a une cohorte à recevoir avec un nombre d'entretiens défini et avec une obligation de résultats à la sortie. Par contre, le contenu de l'accompagnement est à notre main " (directeur, agence Pôle emploi). Tous les conseillers ne développent pas de stratégies de ce type. Comme le rappellent Lipsky (1980) ou Rice (2015), les comportements des agents de terrain face aux politiques publiques qu'ils doivent mettre en œuvre dépendent à la fois du cadre organisationnel (règles, budget, temps, etc.) et de leurs représentations.

Parmi les conseillers rencontrés, près de la moitié ont ainsi expliqué chercher un moyen de travailler avec le prestataire qu'ils jugent le plus pertinent pour l'accompagnement plutôt qu'avec celui imposé par la procédure de marché alors que les autres, généralement plus récemment arrivés en fonction, avaient choisi de se conformer à la procédure de sélection du prestataire. II s'agit pour les premiers, souvent plus expérimentés, de s'appuyer sur leur connaissance du réseau d'acteurs locaux. " $\|$ y a une volonté de renouer avec ces partenaires locaux, j'ai connu ça moi au départ, avec les APP notamment, qui nous ont fait un travail formidable, qu'ont été malheureusement détruits, mais bon... ça revient un petit peu » (conseiller, agence Pôle emploi). Un conseiller raconte ainsi qu'il reçoit un demandeur d'emploi étranger qui a besoin de comprendre la manière dont fonctionne le marché du travail, les pratiques de recrutement et la manière de répondre à une offre d'emploi. Il explique qu'il devrait alors le positionner sur un dispositif traitant de la rédaction du curriculum vitae ou de la recherche d'emploi. Cependant, il peut également choisir un autre dispositif qui n'est pas en lien direct avec la problématique « emploi » du demandeur d'emploi mais dont il sait que le prestataire qui l'accompagnera a une connaissance accrue de ce public et sera mieux à même d'adapter son accompagnement pour répondre à sa problématique individuelle.

Cette stratégie consiste à « bricoler » le système pour reprendre les termes de Garraud (2000) et Farvaque (2011) en faisant reposer le choix d'un prestataire sur ses spécificités plutôt que sur celles de la prestation. Ou pour le dire dans les termes de cette conseillère Pôle emploi, " on joue souvent sur les marges si on veut faire passer des choses. Parce qu'il faut faire financer des formations, si on rentre dans les clous, bien souvent, ça ne se finance pas. Donc on bidouille des trucs à la limite de la réglementation, voire de l'autre côté pour pouvoir financer des choses ". Cet exemple permet de constater que les instruments de marchandisation de l'accompagnement des demandeurs d'emploi, souvent considérés 
comme un frein à l'individualisation, peuvent parfois être contournés par des conseillers expérimentés qui ont une connaissance fine des acteurs locaux et du système de prescription. Cela est rendu possible par la marge de manœuvre dont ils usent et qui demeure en dépit du renforcement d'un nouveau management public souvent assimilé hâtivement à la disparition du pouvoir discrétionnaire des opérateurs de terrain (Evans, Harris, 2004).

\section{Conclusion}

Ainsi, un changement radical (Hall, 1993) a bien eu lieu dans le champ de l'accompagnement des demandeurs d'emploi. En s'appuyant sur la transition d'une définition structurelle à une définition fonctionnelle du SPE, la politique de l'emploi est passée d'un recours segmenté à une externalisation complète du parcours d'accompagnement et de placement des demandeurs d'emploi. Ce changement a été opéré en trois temps par le passage d'une régulation publique monopolistique à la mise en concurrence de l'opérateur public (ANPE) pour finalement s'institutionnaliser dans une externalisation pilotée par celui-ci (Pôle emploi). En se fondant initialement sur des exigences techniques plutôt que sur une plate-forme idéologique, le principe d'un recours à des opérateurs privés de placement est ainsi bien entériné et les instruments de son pilotage ont été expérimentés et mis en place. Pour autant, il serait erroné de croire que ce processus a ouvert la porte à un vaste marché concurrentiel de l'insertion professionnelle qui verrait les opérateurs publics et privés entrer en compétition. Tout d'abord, il faut noter que les volumes d'actions externalisées demeurent modestes et sont plutôt en décroissance. Ensuite, il convient de rappeler que non seulement l'externalisation n'a pas affaibli l'opérateur public - Pôle emploi - mais a en fait renforcé sa position de donneur d'ordre. Par contre, si les effets de cette mise en marché sont modestes au niveau du policy design de la politique de l'emploi, ils sont beaucoup plus substantiels au niveau de sa mise en œuvre concrète.

C'est en effet à ce niveau que l'évolution du cadre réglementaire défie les modes de coopération initialement ancrés dans des relations informelles et/ou historiques et a fait évoluer les relations qu'entretiennent ces acteurs en encourageant un passage d'une relation de partenariat à une relation d'achat de prestation. Ce changement de posture implique un rapport de force entre donneur d'ordre et prestataires qui pèse sur les relations qu'entretiennent les acteurs qui participent au parcours d'insertion professionnelle du demandeur d'emploi. "On travaillait beaucoup avec des partenariats locaux, ce qui n'est plus forcément le cas, où là, les partenaires ou les prestataires répondent à des offres d'emploi, enfin à des appels d'offres, et on les connaît moins quoi » (conseiller, agence Pôle emploi).

De plus, les mécanismes de marchandisation de l'accompagnement, par la logique de volumétrie et la contrainte du prestataire qu'ils sous-tendent, peuvent avoir des effets contreproductifs sur le processus d'individualisation pourtant au cœur de la démarche d'externalisation. Cependant, les conseillers ont une marge de manœuvre leur permettant d'adapter le cadre dans lequel ils évoluent, limitant ainsi le poids des mécanismes de marchandisation de l'accompagnement. Howe prédisait la fin du pouvoir discrétionnaire des street level buraucrats dans les systèmes proches du New Public Management (Howe, 1991). Pourtant, malgré la mise en place de systèmes donnant moins de place à l'intuition du conseiller au 
profit de processus qui leur sont externes, les street level bureaucrats continuent à avoir un rôle majeur dans la manière dont se déploie l'action publique et donc dans la fabrication de l'action publique.

Au final, le mimétisme marchand dans la politique de l'emploi ne semble pas, à ce jour, se manifester par un retrait de l'État de l'accompagnement des chômeurs. En dehors de certaines prestations où le demandeur d'emploi ne rencontre à aucun moment de son accompagnement un agent de Pôle emploi, la plupart des parcours d'insertion professionnelle demeurent portés par cet acteur central du service public de l'emploi qui les réalise en mobilisant ponctuellement des prestataires et reste ainsi encore largement garant de la construction du parcours du demandeur d'emploi. Plutôt qu'un désengagement, la logique de mimétisme marchand aboutit ici à une régulation forte de ce marché d'État et de contrôle des objectifs des nombreux acteurs qui opèrent dans le secteur de l'emploi. La pression tarifaire exercée sur les opérateurs privés mis en concurrence les uns avec les autres aboutit au final à les affaiblir et à abaisser la qualité des prestations offertes aux demandeurs d'emploi (Seibel, 2009 ; Berthet, Conter, 2011; Cour des comptes 2014) plutôt qu'à renforcer un marché privé du placement. Au final donc, l'exemple de la politique de l'emploi montre que, en France, quand l'État singe le marché, ce sont les prestataires privés qui souffrent de la concurrence plus que l'opérateur public.

Thierry Berthet Aix Marseille Univ, CNRS, LEST, Aix-en-Provence, France thierry.berthet@univ-amu.fr

Clara Bourgeois Université de Bordeaux Centre Émile Durkheim brgs.clara@gmail.com

\section{Bibliographie}

\begin{abstract}
AssembléE NATIONALE (2014), Rapport d'information sur le recours aux opérateurs de placement pour l'accompagnement et le placement des demandeurs d'emploi, Commission des finances, de l'économie générale et du contrôle budgétaire, 17 juillet 2014.
\end{abstract}

Balmary, D. (2006), « Un nouveau service public de l'emploi ?", Droit social, 6, p. 594-598.

Balmary, D., Chevrier-Fatome, C., Simonin, B., 2004, Rapport de l'Instance d'évaluation de la politique d'emploi et recours à des opérateurs externes, Paris, La Documentation française.

Béraud, M., Eydoux, A. (2009), " Activation des chômeurs et modernisation du service public de l'emploi ", Travail et emploi, 119, juillet-septembre 2009, mis en ligne le 30 septembre 2011 [http://travailemploi.revues.org/3468] (consulté le 2 novembre 2015).
Berthet, T. (2010), «Externalisation et gouvernance territoriale des politiques de l'emploi ", Revue française de socio-économie, 6, p. 131-148.

BERTHET, T. (2012), « Les politiques régionales de formation professionnelle : quelques lignes de changement ", Schedae 3, Presses universitaires de Caen, p. 33-44.

Berthet, T., Bourgeois, C. (2016), "The Effects of Local Target Group Specifications on Activation: The Case of France ", in HeIDENREICH, M., RICE, D. (eds), Integrating Social and Employment Policies in Europe: Active Inclusion and Challenges for Local Welfare Governance, Cheltenham, Edward Elgar.

Berthet, T., Conter, B. (2011), " Les changements d'instruments de la politique de l'emploi en Wallonie et en France ", Travail et emploi, 125, janvier-mars, p. 55-65. 
Bessy, C., Eymard-Duvernay, F. (dir.) (1997), Les Intermédiaires du marché du travail, Paris, PUF.

Bezes, P., Demazière, D., Le Bianic, T., Paradeise, C., Normand, R., Benamouzig, D., Pierru, F., Evetts, J. (2011), " New Public Management et professions dans l'État : au-delà des oppositions, quelles recompositions ? ", Sociologie du travail, 53, p. 293-348.

Bourgeols, C. (2015), Les Défis de l'intersectorialité : l'exemple de la mise en œuvre des dispositifs d'insertion professionnelle des immigrés, thèse de science politique, Bordeaux, Université de Bordeaux.

Chevallier, J. (2005), « Politiques publiques et changement social», Revue française d'administration publique, 115, p. 383-390.

Colomb, F. (2012), Les Politiques de l'emploi (1960-2000). Sociologie d'une catégorie de politique publique, Rennes, Presses universitaires de Rennes.

Considine, M. (2001), Enterprising States. The Public Management of Welfare to Work, Cambridge (Mass.), Cambridge University Press.

CONTER, B. (2015), La Stratégie européenne pour l'emploi, ses usages et effets en Wallonie, thèse de doctorat en science politique, Bordeaux, IEP de Bordeaux.

COUR DES COMPTES (2014), Le Recours par Pôle emploi aux opérateurs privés pour l'accompagnement et le placement des demandeurs d'emploi, Paris, Cour des comptes, mai.

COUR DES COMPTES (2015), Pôle emploi à l'épreuve du chômage de masse, Paris, Cour des comptes, juillet.

Crozier, M., Friedberg, E. (1977), L'Acteur et le Système, Paris, Seuil.

Cuntigh, P., Perez, C., Personnaz, E. (2008), Les Services d'information, de conseil et d'orientation professionnelle des adultes : un appui aux transitions professionnelles ?, Marseille, CEREQ, coll. "Relief », 27.

Devaux-Spatarakis, A. (2014), La Méthode expérimentale par assignation aléatoire : un instrument de recomposition de l'interaction entre sciences sociales et action publique en France?, thèse de doctorat en science politique, Bordeaux, IEP de Bordeaux.

Diemer, A., Guillemin, H. (2009), "La marchandisation du travail dans la société anglaise de la fin du XVII" siècle au début du XIX siècle ", Cahiers d'histoire. Revue d'histoire critique, 110, p. 53-81.

DivaY, S. (2009), "Nouveaux opérateurs privés du service public de l'emploi », Travail et emploi, 119, juillet-septembre, p. 37-49.

DuBoIs, V. (2013), « Le rôle des street level bureaucrats dans la conduite de l'action publique en France [halshs00660673], [https://halshs.archives-ouvertes.fr/halshs- 00660673/document] (consulté le 11 septembre 2017).

Evans, T., Harris, J. (2004), "Street-Level Bureaucracy, Social Work and the (Exaggerated) Death of Discretion ", British Journal of Social Work, 34 (6), p. 871-895.

FARVAQUE, N. (2011), « Le bricolage du maintien dans l'emploi des seniors: régulation publique, dialogue social et boîte à outils ", La Revue de l'Ires, 69 (2), p. 139-172.

Ferracci, M. (2007), "Améliorer le service public de l'emploi : ce que disent les faits ", Revue française d'économie, 21 (3), p. 75-135.

FOURNIER, P. (2000), «Éléments pour une histoire des textes concernant le placement et l'embauche des travailleurs ", extraits des Cahiers du CHATEFP, 2-3, janvier 2000 [http://travail-emploi.gouv.fr/IMG/pdf/ Elements_pour_une_histoire_des_textes_concernant _le_placement_et_I_embauche_des_travailleurse-2.pdf] (consulté le 11 septembre 2017).

Galbraith, J.-K. (1997), « Dangerous Metaphor: The Fiction of the Labor Market. Unemployment, Inflation, and the Job Structure", Policy Brief, $\mathrm{n}^{\circ} 36$, The Jerome Levy Economics Institute of Bard College.

Garraud, P. (2000), Le Chômage et l'action publique: le "bricolage» institutionnalisé, Paris, L'Harmattan, coll. "Logiques politiques ».

Gelot, D., Nivolle, P., Bompart, J.-P. (dir.) (2000), Les Intermédiaires des politiques publiques de l'emploi, Paris, La Documentation française, coll. "Cahiers Travail et emploi ».

Georges, N. (2007), "L'externalisation de l'accompagnement des demandeurs d'emploi: modalités d'un marché en plein essor ", Centre d'études de l'emploi, document de travail $n^{\circ} 81$.

Green, I., Doellgast, V. (2017), « Marketization, Inequality and Institutional Change: Toward a New Framework for Comparative Employment Relations", Journal of Industrial Relations, 59 (2), p. 192-208.

HALL, P. (1993), « Policy Paradigms, Social Learning, and the State, The Case of Economic Policymaking in Britain ", Comparative Politics, 25, p. 274-296.

Hisou, B. (1998), "Retrait ou redéploiement de l'État? ", Critique internationale, 1, p. 151-168.

Howe, D. (1991), "Knowledge, Power and the Shape of Social Work Practice ", in DAviES, M. (ed.), The Sociology of Social Work, Londres, Routledge, p. 202-220.

Lascoumes, P., Le Galès, P. (2004), Gouverner par les instruments, Paris, Presses de Sciences Po.

LAVITRY, L. (2010), « Rationalisation et individualisation dans les services publics : le cas des conseillers à l'emploi ", dans JACQUOT, L. (dir.), Formes et structures du salariat: crise, mutation, devenir, Nancy, 
Presses universitaires de Nancy, coll. "Salariat et transformations sociales », p. 135-147.

LE GALÈs, P., Scott, A. (2008), « Une révolution britannique? Autonomie sans contrôle ou "freer markets, more rules" ", Revue française de sociologie, 49 (2), p. 301-330.

LIPSKY, M. (1980), Street-Level Bureaucracy: Dilemmas of the Individual in Public Services, New York (N. Y.), Russell Sage Foundation.

Mathiot, P. (2000), Acteurs et politiques de l'emploi en France (1981-1993), Paris, L'Harmattan.

MerRien, F.-X. (1999), "La nouvelle gestion publique : un concept mythique ", Lien social et politiques, 41, p. 95-103.

Ministère des AfFaIRes ÉtRAngères et du DÉVELopPeMENT INTERNATIONAL (2015), Étude d'impact, Projet de loi autorisant la ratification de la convention $n^{\circ} 181$ de l'Organisation internationale du travail relative aux agences d'emploi privées [www.assemblee-nationale. fr/14/projets/pl1887-ei.asp] (consulté le 2 novembre 2015].

MULLER, M. (1990), « La naissance de l'agence nationale pour l'emploi. Institution et mission de service public ", Sociétés contemporaines, 3 (1), p. 19-32.

MULleR, P. (2015), La Société de l'efficacité globale. Comment les sociétés modernes se pensent et agissent sur elles-mêmes, Paris, PUF.

Muller, P., SuRel, Y. (1998), L'Analyse des politiques publiques, Paris, Montchrestien, coll. "Clefs ».

PARENT, G., SAUtory, O. et al. (2014), « L'accompagnement des demandeurs d'emploi : effets du renforcement et de l'externalisation du suivi ", Travail et emploi, 139, p. 75-89.

PolAnYI, K. (2011), La Subsistance de l'homme dans l'histoire et la société, Paris, Flammarion.

PÔLE EMPLOI (2012), "Le recours aux opérateurs privés de placement. Les résultats de l'étude qualitative menée en 2011 ", Repères et analyses, 37.

Pôle EMPLOI (2015), Réussir ensemble [En ligne], [www.poleemploi.org/file/galleryelement/pj/26/71/9d/ ab/pe2015--v2-cce-du 5970696908407599716.pdf] (consulté le 15 novembre 2014).

Postel, N., Sobel, R. (2010), «La RSE : nouvelle forme de dé-marchandisation du monde? Une lecture institutionnaliste à partir de Karl Polanyi ", Développement durable et territoires [En ligne], 1 (3), décembre, mis en ligne le 8 janvier 2013, [http:// developpementdurable.revues.org.inshs.bib.cnrs.fr/ 8506;DOI:10.4000/developpementdurable.8506] (consulté le 7 septembre 2017).
Pressman, J. L., Wildavsky, A. B. (1984 [1973]), Implementation: How Great Expectations in Washington Are Dashed in Oakland; Or, Why It's Amazing that Federal Programs Work at All, This Being a Saga of the Economic Development Administration as Told by Two Sympathetic Observers Who Seek to Build Morals on a Foundation of Ruined Hopes, Berkeley (Calif.), University of California Press.

RICE, D. (2015), Building Welfare States. How Policy Shapes Caseworker Practice?, Amsterdam, VU University Press.

SEIBEL, C. (2009), Rapport de synthèse du comité de pilotage de l'évaluation sur les expérimentations l'accompagnement renforcé des demandeurs d'emploi conduites par l'Unédic et l'ANPE en 2007, Paris, octobre.

SoldinI, D. (2006), "Le service public de l'emploi et le droit de la concurrence", Droit social, 6, p. 599-601.

ToRdJman, H. (2004), " How to Study Markets? An Institutionalist Point of View », Revue d'économie industrielle, 107 (1), p. 19-36.

Van Berkel, R., GraAf, W. de, Sirovatka, T. (2012), "Governance of Activation Policies in Europe ", International Journal of Sociology and Social Policy, 32 (5), p. 260-272.

Van Berkel, R., Valkenburg, B. (2004), Making it Personal: Individualising Activation Services in the EU, Bristol, The Policy Press.

VATAN, S. (2013), "Faut-il rompre avec les quasimarchés pour penser la marchandisation ? ", communication au $3^{e}$ congrès de l'AFEP, Bordeaux, juillet [http://afep2013.gretha.u-bordeaux4.fr/IMG/ pdf/vatan_-_afep2013.pdf] (consulté le 4 novembre 2015).

VIVES, C. (2013), L'Institutionnalisation du recours aux opérateurs privés de placement au cœur des conflits de régulation du service public de l'emploi ? (2003-2011), thèse de sociologie Nanterre, Université de Paris-Ouest-Nanterre, novembre.

VIVES, C. (2014), Un enjeu au cœur des transformations du service public de l'emploi: externaliser le placement, CEE, Connaissance de l'emploi, 110, février.

VIVES, C. (2017), "L'évaluation randomisée dans la réforme du service public de l'emploi ", Noisy le Grand, CEET, Documents de travail, 190.

Zimmermann, K., Aurich, P., Graziano, P., Fuertes, V. (2014), " Local Worlds of Marketization. Employment Policies in Germany, Italy and the UK Compared", Social Policy \& Administration, 48 (2), p. 127-148. 